\title{
Correction to: Cardiac Magnetic Resonance Imaging in Pulmonary Arterial Hypertension: Ready for Clinical Practice and Guidelines?
}

\section{Barbro Kjellström ${ }^{1,2} \cdot$ Anthony Lindholm ${ }^{1} \cdot$ Ellen Ostenfeld ${ }^{1}$}

Published online: 14 September 2020

(C) Springer Science+Business Media, LLC, part of Springer Nature 2020

\section{Correction to: Current Heart Failure Reports (2020) 17:181-191 https://doi.org/10.1007/s11897-020-00479-7}

The original version of this article contains error in the text.

The text currently reads:

Reduced strain increases the risk for adverse events such as death, lung transplant, and functional class deterioration, incurring a hazard ratio (HR) of 1.06 for LV longitudinal strain, 2.52 for RV longitudinal strain, and even as much as 4.5 for RV circumferential strain [28].

The corrected text is as follows:

Reduced strain in the RV increases the risk for adverse events such as death, lung transplant, and functional class deterioration, incurring a hazard ratio (HR) of 1.06 for RV longitudinal strain, 2.52 for RV longitudinal strain rate, and even as much as 4.5 for RV circumferential strain [28].

Publisher's Note Springer Nature remains neutral with regard to jurisdictional claims in published maps and institutional affiliations.

The online version of the original article can be found at https://doi.org/ 10.1007/s11897-020-00479-7

Ellen Ostenfeld

ellen.ostenfeld@med.lu.se

Barbro Kjellström

barbro.kjellstrom@ki.se

Anthony Lindholm

anthony.lindholm@med.lu.se

1 Department of Clinical Sciences Lund, Clinical Physiology and Skåne University Hospital, Lund University, SE-221

85 Lund, Sweden

2 Swedish Pulmonary Arterial Hypertension Registry, Uppsala Clinical Research Centre, Uppsala University, Uppsala, Sweden 